\title{
Orbital period changes and the higher-order multiplicity fraction amongst SuperWASP eclipsing binaries *
}

\author{
M. E. Lohr ${ }^{1}$, A. J. Norton ${ }^{1}$, S. G. Payne ${ }^{1}$, R. G. West ${ }^{2}$, and P. J. Wheatley ${ }^{2}$ \\ 1 Department of Physical Sciences, The Open University, Walton Hall, Milton Keynes MK7 6AA, UK \\ e-mail: Marcus.Lohr@open.ac.uk \\ 2 Department of Physics, University of Warwick, Coventry CV4 7AL, UK
}

Received 26 January 2015 / Accepted 29 April 2015

\begin{abstract}
Orbital period changes of binary stars may be caused by the presence of a third massive body in the system. Here we have searched the archive of the Wide Angle Search for Planets (SuperWASP) project for evidence of period variations in 13927 eclipsing binary candidates. Sinusoidal period changes, strongly suggestive of third bodies, were detected in $2 \%$ of cases; however, linear period changes were observed in a further $22 \%$ of systems. We argue on distributional grounds that the majority of these apparently linear changes are likely to reflect longer-term sinusoidal period variations caused by third bodies, and thus estimate a higher-order multiplicity fraction of $24 \%$ for SuperWASP binaries, in good agreement with other recent figures for the fraction of triple systems amongst binary stars in general.
\end{abstract}

Key words. stars: variables: general - binaries : close - binaries: eclipsing

\section{Introduction}

The importance of multiplicity for an understanding of stellar evolution is hard to overestimate. Single stars now appear to be in a minority, especially at higher masses (the recent review of Duchêne \& Kraus 2013 indicated a multiplicity fraction for intermediate-mass stars $\geq 50 \%$, rising to $\geq 80 \%$ for the most massive stars), and binary interactions are probably responsible for creating several different types of supernovae, novae and unusual star types such as blue stragglers. Amongst binaries, a significant proportion appear to be part of higher-order multiple systems (Tokovinin 2014a,b, estimated $29 \%$ for F and G dwarfs in a distance-limited sample), and such triples, quadruples etc. also have much to tell us about the formation and stability of stellar systems (see e.g. Michaely \& Perets 2014; Naoz \& Fabrycky 2014).

Higher-order multiple star systems can be detected by a range of methods including direct resolution, radial velocity and proper motion analysis. In several recent papers, we have used archive light curves from the Wide Angle Search for Planets (SuperWASP; Pollacco et al. 2006) to study eclipsing binaries, benefiting from its high-cadence, long-baseline time-domain photometry. With such data, additional eclipses in a binary light curve may occasionally reveal further bodies in a system, as with the doubly-eclipsing quintuple system reported in Lohr et al. (2013b) and followed up by Koo et al. (2014). However, a more widely-applicable technique will be the detection of eclipse timing variations producing an approximately sinusoidal curve in an $\mathrm{O}-\mathrm{C}$ (observed minus calculated) diagram, as in Lohr et al. (2013a), where a triple system containing an M+M contact binary was proposed on such evidence, and later confirmed by

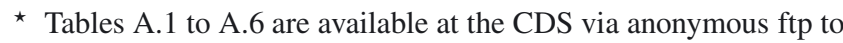
cdsarc.u-strasbg.fr $(130.79 .128 .5)$ or via

http://cdsarc.u-strasbg.fr/viz-bin/qcat?]/A+A/578/A136
Koen (2014), in Lohr et al. (2014) also, period variations arguably produced by circumbinary planets were detected in several post-common-envelope binaries. This approach was used by Rappaport et al. (2013) to search for candidate triples in the Kepler eclipsing binary catalogue. Here, we use a preliminary catalogue of candidate SuperWASP eclipsing binaries to search for orbital period changes potentially indicative of third bodies. These statistics can then be used to suggest a lower limit to the higher-order multiplicity fraction of SuperWASP stars.

\section{Method}

A provisional catalogue of SuperWASP eclipsing binary candidates was produced by Payne (2013), using a neural net classification method for all objects listed in the database with periods found by the method described in Norton et al. (2007, 2011). The catalogue contained 2875 objects classified as EWtype (probable contact) binaries, 5226 EB-type (light curve resembling $\beta$ Lyrae), 5826 EA-type (light curve resembling Algol $=\beta$ Persei) exhibiting two eclipses per cycle, and 7056 potential EA-type systems in which only a single eclipse was visible. Owing to the large number of false positives expected in the last group, only the first three groups of sources were considered here for further analysis.

13927 light curves were downloaded from the SuperWASP archive, and a form of the custom IDL code described in Lohr et al. (2014), modified for large numbers, was run on them. This checked and refined the orbital period associated with each object identifier, searching within a range centered on the catalogue period (itself derived from the archive database); produced a phase-folded light curve and mean fitting curve (with 100 bins); generated $\mathrm{O}-\mathrm{C}$, amplitude change and absolute flux change diagrams; and determined a rate of period change where this was supported by the $\mathrm{O}-\mathrm{C}$ diagram. The output for each identifier was an image file allowing visual checking of the light curve, 


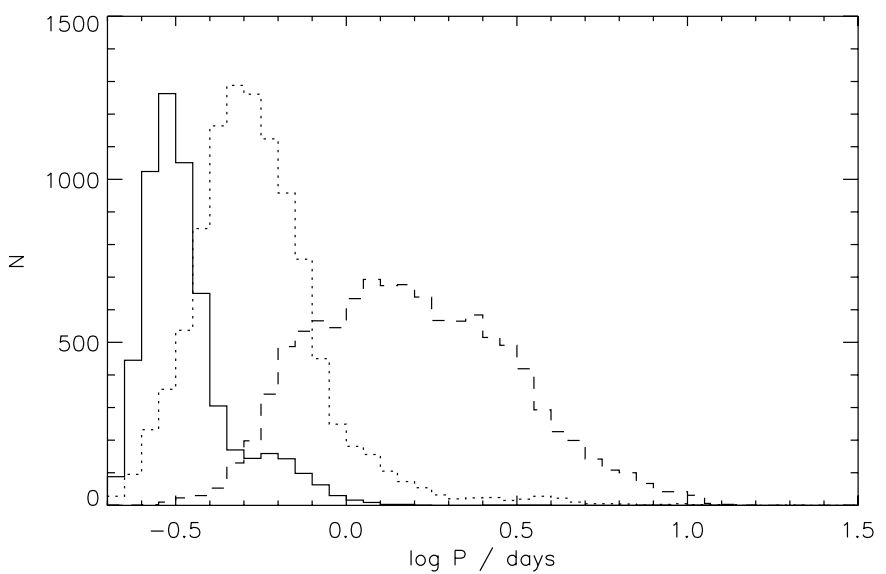

Fig. 1. Period distributions of SuperWASP eclipsing binary candidates after preliminary quality checks. EW-type objects are shown with a solid line (cf. Paczyński et al. 2006 EC group); EBs with a dotted line (cf. Paczyński et al.'s ESD group); EAs with a dashed line (cf. Paczyński et al.’s ED group).

$\mathrm{O}-\mathrm{C}$ and other diagrams; and a log file line summarizing key statistics such as number of data points in the light curve, mean flux, orbital period and evidence for period change.

The $\log$ file revealed a number of objects clustered near particular periods: $1 / 4,1 / 3,2 / 5$ and $2 / 3$ of a sidereal day in particular. Visual checks of these objects (mainly on the EA list) confirmed that they were spurious periodic variables, with the variability probably resulting from temperature-related instrumental effects occurring daily e.g. peaks or troughs in the light curve at the start of each night's observations. These identifiers were removed from the list. For some objects, no significant period could be found in the given range, or no $\mathrm{O}-\mathrm{C}$ diagram could be constructed (due to insufficient successful fits to nightly observations), and these objects were also removed from further consideration. These preliminary checks left 2844 EW-type, 5073 EB-type and 5323 EA-type objects.

Figure 1 shows their period distribution by type; a broadly comparable collection from the ASAS database is given in Paczyński et al. (2006) Fig. 6. Although our collection contains proportionally far fewer contact-type binaries (or perhaps far more of the other two types), the peaks and ranges of the distributions are very similar: EWs peak around $P=0.3-0.35 \mathrm{~d}$ and tail off above about $1 \mathrm{~d}$; EBs peak around $P=0.5 \mathrm{~d}$ and are very rare beyond $2 \mathrm{~d}$; both types drop off sharply in period below the "short period cut-off" at around $P=0.20 \mathrm{~d}$ (see Lohr et al. 2012, 2013b, for more detail on the period distribution of SuperWASP eclipsing binaries in this region). EAs have a broader peak around $P=1-3 \mathrm{~d}$, and are very rare below $P=$ $0.3 \mathrm{~d}$ and above $P=10 \mathrm{~d}$. Catalogues of specific types of eclipsing binaries, such as classical Algols (see Budding et al. 2004; Ibanoglu et al. 2006 and especially van Rensbergen et al. 2011, Fig. 5), exhibit greater numbers at longer periods; however, these are less likely to be detected reliably in SuperWASP data due to time-sampling limitations or because they are brighter than $V \sim 9 \mathrm{mag}$.

For the remaining objects, where period change had been found by the code, the ratio between best linear and quadratic fit reduced $\chi^{2}$ values for the $\mathrm{O}-\mathrm{C}$ diagram was used to select a sample for visual checking. All output files were checked down to a ratio of 1.25 , below which it was generally difficult to judge the classification reliably by eye; tests applied to eclipsing post-common-envelope binaries in Lohr et al. (2014) had also indicated that ratios below 1.05 did not generally indicate statistically significant period change. This meant that $679 \mathrm{EW}$ type, 436 EB-type and 806 EA-type objects were checked visually, and assigned a classification: plausible quadratic variation in the $\mathrm{O}-\mathrm{C}$ diagram (supporting secular period change); plausible sinusoidal variation (supporting alternating period increases and decreases); no apparent period change (usually due to erratic time sampling misleading the program's fitting algorithm); erroneous period found (usually due to the original input period being significantly wrong); or unclear (usually when the time sampling was very sparse or the time basis very limited).

\section{Results}

Period change was indicated by our code for 2305/2844 EWtype, 3227/5073 EB-type and 3076/5323 EA-type objects. However, these fractions cannot be taken at face value: large numbers of these apparent changes involved very small differences between the best linear fit and the best quadratic fit to the $\mathrm{O}-\mathrm{C}$ diagram, which would probably not have been statistically significant; many of the EA-type objects exhibiting apparent period change also turned out to have erroneous periods - usually those with very long periods which were poorly sampled - and this could create the illusion of quadratic period change.

480/679 EW-type visually-checked objects were classified as exhibiting plausible period change, of which 388 showed quadratic and 92 sinusoidal behaviour (Tables A.1 and A. 2 give a complete list). Figure 2 illustrates a clear case of period increase in this type. Extending this proportion to all the objects with linear-quadratic fit ratios above 1.05, and adjusting the whole sample size to account for expected numbers of erroneous periods and uncertain cases, we can estimate that about $41 \%$ of the EW-type objects are undergoing period change (see Table 1 for the full figures used in this calculation).

$167 / 436$ EB-type objects were classified as exhibiting plausible period change, 137 quadratic and 30 sinusoidal (Tables A.3 and A.4). Figure 3 illustrates secular period change in this type, while Fig. 4 shows very clear sinusoidal variation (though this might be better described as an EW-type system). Scaling up the numbers as before, we can estimate that about $19 \%$ of the EB-type objects are undergoing period change.

189/806 EA-type objects were classified as exhibiting plausible period change, 172 quadratic and 17 sinusoidal (Tables A.5 and A.6). Figure 5 shows a probable case of unusually short-term sinusoidal variation in this type. Scaling up the numbers as before, we can estimate that about $14 \%$ of the EA-type objects are undergoing period change.

Of the $\mathrm{O}-\mathrm{C}$ diagrams judged to be exhibiting sinusoidal variation, most ( $\sim 70 \%$ for EW-type; $\sim 90 \%$ for EBs and EAs) show approximately one complete cycle (as in Fig. 4), with nearequal proportions of such cases starting with period increase or decrease i.e. there does not seem to be a detection bias in favour of either. Since sinusoidal fitting could not be reliably automated for these (often noisy) data sets, best-fit modulation periods were not obtained for the majority of objects, but visual estimation suggested that the binaries with shorter orbital periods (mostly EW-type) tend to possess shorter modulation periods. This probably explains why around $30 \%$ of the sinusoidally-varying EWs show more than one complete cycle during their time of observation by SuperWASP, while only about $10 \%$ of EBs and EAs do; Fig. 6 illustrates one such case where three complete cycles have arguably been captured. 
M. E. Lohr et al.: Period changes and multiplicity in SuperWASP binaries

Table 1. Object statistics used in multiplicity calculations.

\begin{tabular}{lllll}
\hline \hline & & EW-type & EB-type & EA-type \\
\hline 1 & Total objects considered & 2844 & 5073 & 5323 \\
2 & Period change found by code & 2305 & 3227 & 3076 \\
3 & ${\text { Objects with } \chi^{2} \text { ratio } \geq 1.05^{a}}_{1414}$ & 1395 & 1797 \\
4 & Objects without apparent period change $^{b}$ & 1430 & 3678 & 3526 \\
5 & Objects checked visually $_{6}$ & 679 & 436 & 806 \\
6 & Period change confirmed visually & 480 & 167 & 189 \\
7 & Period change rejected visually & 88 & 50 & 48 \\
8 & Wrong period detected visually & 24 & 33 & 340 \\
9 & Unclear cases on visual check $_{10}$ & 87 & 186 & 229 \\
\hline 10 & Corrected count of genuine period changes $^{c}$ & 1000 & 534 & 421 \\
11 & Corrected count of wrong periods $s^{d}$ & 50 & 106 & 758 \\
12 & Corrected count of unclear cases & \\
13 & Corrected count of valid objects & 364 & 2164 & 1512 \\
\hline 14 & Corrected period change percentage $^{g}$ & 2430 & 2803 & 3053 \\
\hline
\end{tabular}

Notes. ${ }^{(a)}$ See Lohr et al. (2014) for explanation of this ratio. ${ }^{(b)}$ I.e. row 1 - row 3. ${ }^{(c)}$ Obtained by extending the confirmed period change ratio (row 6/row 5) to all objects with plausibly-significant $\chi^{2}$ ratios (row 3). ${ }^{(d)}$ Obtained by extending the detected wrong period ratio (row 8/row 5) to all objects with plausibly-significant $\chi^{2}$ ratios (row 3). ${ }^{(e)}$ Obtained by extending the unclear cases ratio (row 9/row 5) to all objects considered (row 1). ${ }^{(f)}$ I.e. total objects considered minus expected objects with wrong periods or unclear cases (row $1-($ row $11+$ row 12$)$ ). ${ }^{(g)}$ I.e. (row $10 /$ row 13$) \times 100 \%$.
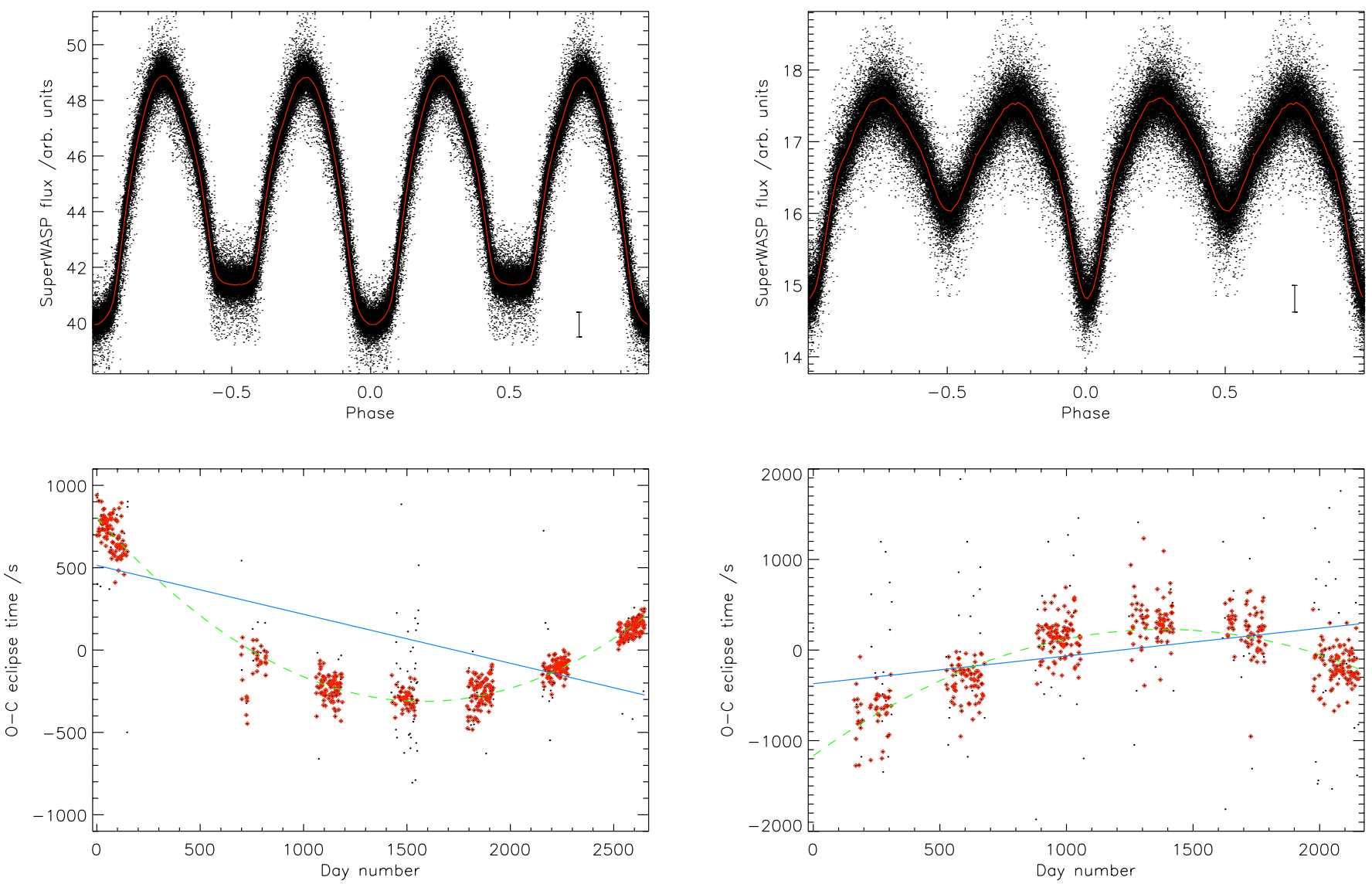

Fig. 2. Top: light curve for J171747 (automatically classified as EWtype) folded at $P=38649.224 \pm 0.006 \mathrm{~s}$, with binned mean curve overplotted in red (colour version in online edition only). A representative error bar for a single observation is shown in the lower corner. Bottom: $\mathrm{O}-\mathrm{C}$ diagram for J171747 spanning eight years. Red (larger) points were automatically selected for period change determination; black (smaller) points were excluded as outliers (a few additional more extreme outliers fall outside the bounds of the plot). Blue solid line shows best linear fit (reduced $\chi^{2}=13.58,548$ degrees of freedom); green dashed line shows best quadratic fit $\left(\chi^{2}=1.03,547\right.$ d.o.f.), strongly supporting a secular period increase, with rate $0.1466 \pm 0.0018 \mathrm{~s} \mathrm{yr}^{-1}$.

Fig. 3. Top: light curve for J064024 (automatically classified as EBtype) folded at $P=44851.164 \pm 0.007$ s. Bottom: $\mathrm{O}-\mathrm{C}$ diagram for J064024 covering six years. Blue solid line shows best linear fit $\left(\chi^{2}=2.96,466\right.$ d.o.f. $)$; green dashed line shows best quadratic fit $\left(\chi^{2}=1.01,465\right.$ d.o.f.), strongly supporting a secular period decrease, with rate $-0.277 \pm 0.009 \mathrm{~s} \mathrm{yr}^{-1}$.

Plotting (quadratic) period change measurements against periods (Fig. 7) for the three types of eclipsing systems, we may note that the most rapid changes are found in the long-period 

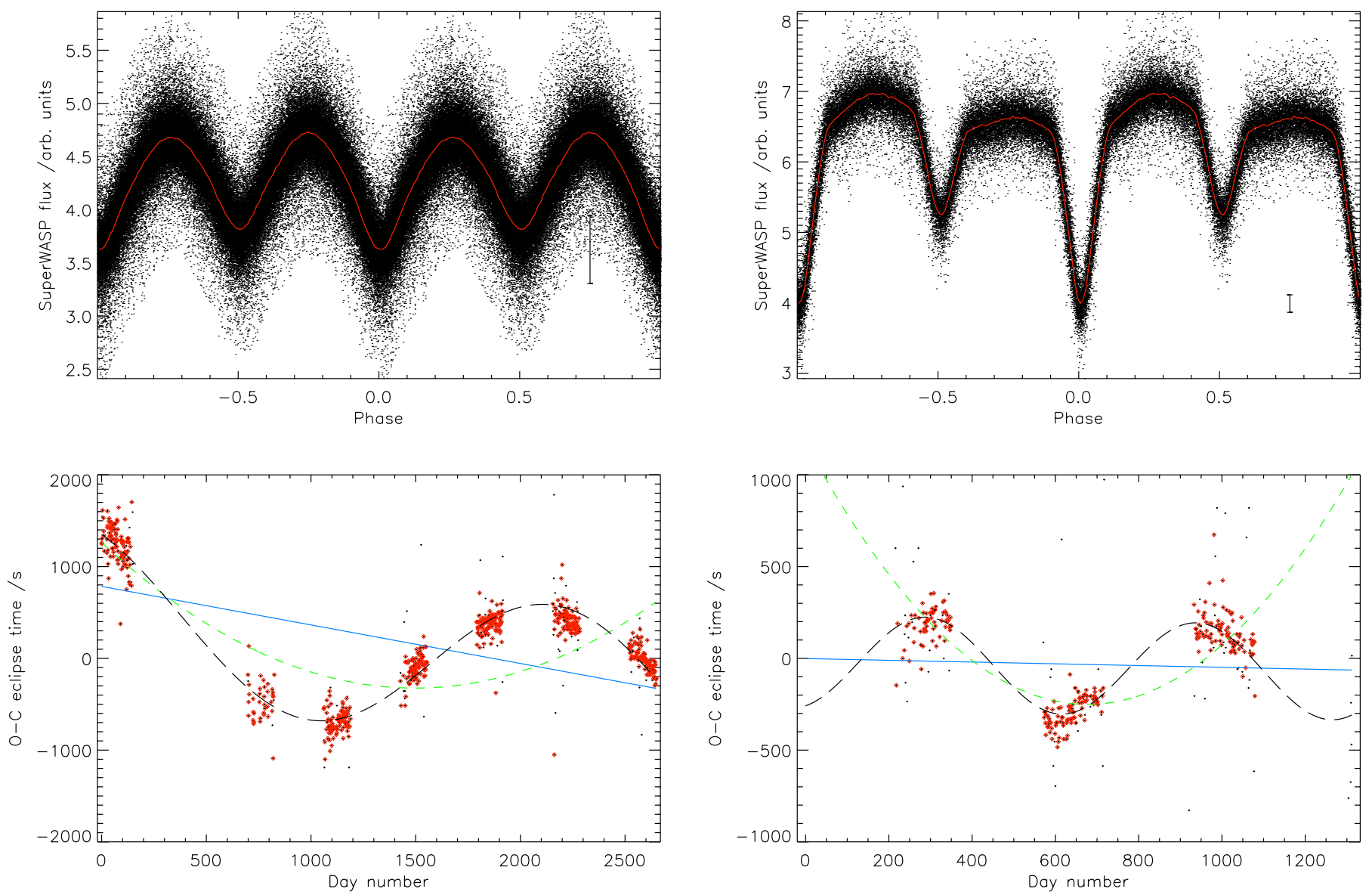

Fig. 4. Top: light curve for J165649 (automatically classified as EBtype) folded at $P=23775.304 \pm 0.003 \mathrm{~s}$. The short period and light curve shape would probably support EW-type classification instead. Bottom: O-C diagram for J165649 spanning eight years. Blue solid line shows best linear fit $\left(\chi^{2}=4.51,582\right.$ d.o.f.); green dashed line shows best quadratic fit $\left(\chi^{2}=2.14,581\right.$ d.o.f.), which are similarly poor matches to the data. Black long-dashed line shows best sinusoidal fit $\left(\chi^{2}<1.00\right.$ using same $\mathrm{O}-\mathrm{C}$ uncertainties as for linear and quadratic fits, 580 d.o.f.), with semi-amplitude $870 \pm 9 \mathrm{~s}$ and modulation period $2386 \pm 11 \mathrm{~d}$.

EA-type binaries, while the short-period EWs all have period changes below $1 \mathrm{~s} \mathrm{yr}^{-1}$ in magnitude. The shortest $P / \dot{P}$ time scale (i.e. a time to merger, if the period decrease continued at this rate) is seen in EB/EW-type object J051927, at 24 $000 \mathrm{yr}$.

Nearly equal proportions of period increases $(51.6 \%)$ and decreases (48.4\%) are found, without significant differences between the three types. Moreover, the period change distributions for the three types (Figs. 8 to 10) are very similar in shape: peaked strongly at small values on each side of zero, with approximately Gaussian tails at larger values, and a gap around zero itself, where genuine period changes are very hard to detect. A Kolmogorov-Smirnov test of the positive values of each distribution against the negative values does not support any significant difference between them i.e. they are symmetric in a statistical sense. However, when all three distributions are considered simultaneously, the K-S test provides near-significant support for the two sides being drawn from different underlying distributions $(P=0.07)$, and we may note that the peak on the positive side is slightly higher than the peak on the negative side in all three histograms.

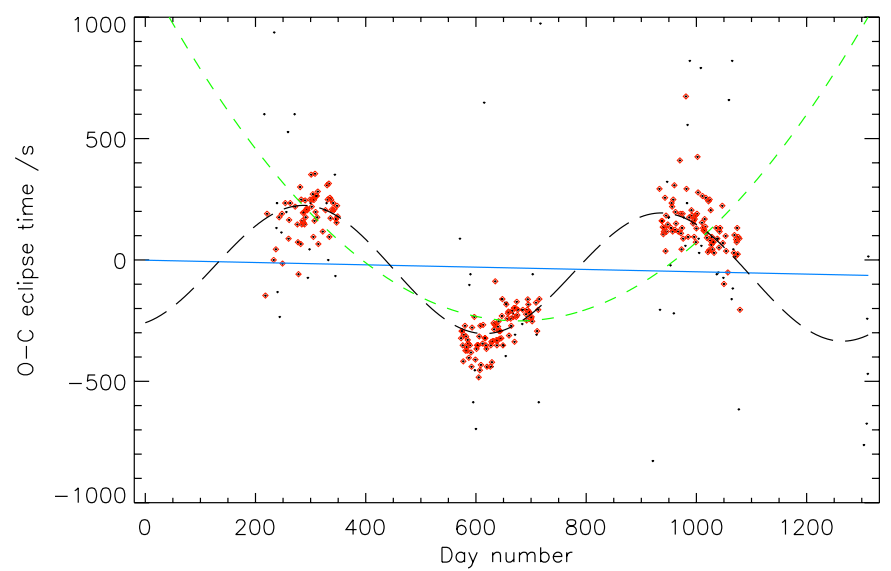

Fig. 5. Top: light curve for J161253 (automatically classified as EAtype) folded at $P=35162.816 \pm 0.007$ s. Bottom: O-C diagram for J161253 covering just over three years. Blue solid line shows best linear fit $\left(\chi^{2}=4.15,235\right.$ d.o.f.); green dashed line shows best quadratic fit $\left(\chi^{2}=1.10,234\right.$ d.o.f.). Although a reasonable quadratic fit to the first three years of data has been achieved, the $\mathrm{O}-\mathrm{C}$ trends within each year and the location of the partial data from year 4 on the diagram (small points around day 1300) lend more support to a sinusoidal variation. Black dotted line shows best sinusoidal fit $\left(\chi^{2}<1.00\right.$ using same $\mathrm{O}-\mathrm{C}$ uncertainties as for linear and quadratic fits, 233 d.o.f.), with semiamplitude $256 \pm 6 \mathrm{~s}$ and modulation period $649 \pm 9 \mathrm{~d}$.

\section{Discussion}

As was found in Lohr et al. (2013b), it is notable that the (quadratic) period change distributions found here are all broadly symmetric, with binary systems of all three light curve classes apparently as likely to increase in orbital period as to decrease. The three distributions are also similar in shape, differing primarily in scale (the longer the orbital period, the greater the typical rate of period change, so that $\dot{P} / P$ is roughly constant). This symmetry would not necessarily be expected given the usual model of W UMa-type contact binaries forming ultimately from low-mass detached systems as they move from wider to smaller separations, primarily due to magnetic braking (e.g. Hilditch 2001; Stepień \& Gazeas 2012); indeed, if there is a slight asymmetry in Figs. 8 to 10, it is in the direction of period increase rather than decrease. Moreover, such evolution by magnetic braking would normally be expected to be two or three orders of magnitude slower than the changes measured here: Eggleton (2006) tentatively suggests a binary composed of Solar-type stars might decrease in period from a few days to contact ( $\sim 0.3$ days) in something like the Hubble time. More 
M. E. Lohr et al.: Period changes and multiplicity in SuperWASP binaries
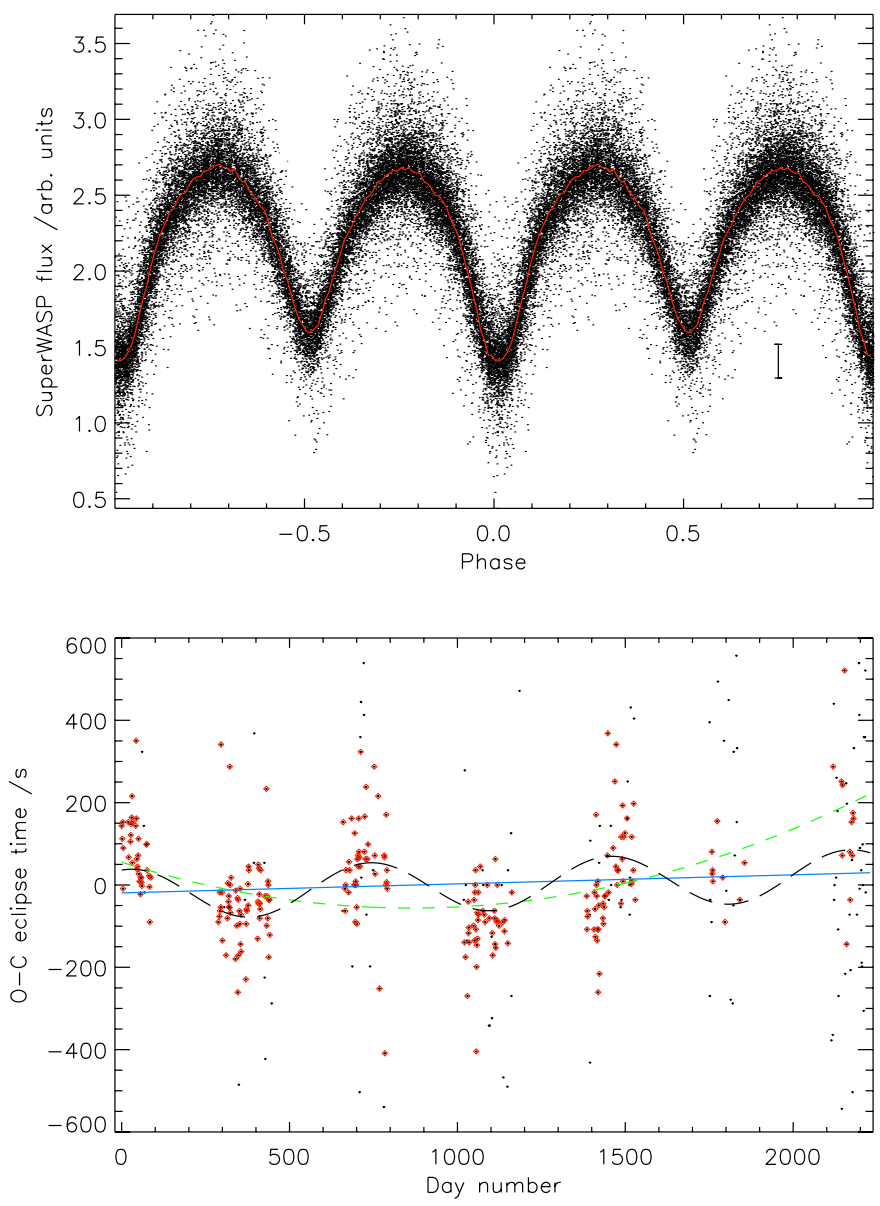

Fig. 6. Top: light curve for J142508 (automatically classified as EWtype) folded at $P=21568.141 \pm 0.002 \mathrm{~s}$. Bottom: O-C diagram for J142508 spanning seven years. Blue solid line shows best linear fit $\left(\chi^{2}=1.28,252\right.$ d.o.f. $)$; green dashed line shows best quadratic fit $\left(\chi^{2}=1.01,251\right.$ d.o.f.). Visual checks revealed that the apparently good quadratic fit relies in part on the widely-scattered poorer-quality data in the last two observing seasons, and appears inconsistent with the bulk of the closely-grouped observations in the third season (around days 700-800); a sinusoidal fit was therefore preferred. Black long-dashed line shows best sinusoidal fit $\left(\chi^{2}<1.00\right.$ using same $\mathrm{O}-\mathrm{C}$ uncertainties as for linear and quadratic fits, 250 d.o.f.), with semi-amplitude $62 \pm 7 \mathrm{~s}$ and modulation period $710 \pm 50 \mathrm{~d}$.

massive Algol-type systems are currently modelled (Siess et al. 2013; Deschamps et al. 2013) as undergoing initial period decrease associated with mass transfer, followed by a substantial period increase after mass ratio reversal; however, the majority of SuperWASP objects may be expected to be relatively lowmass (the survey's magnitude limits mean that it mainly detects sources in the local volume of the Milky Way, in which lower-mass stars predominate). In any case, mass-transfer evolution would not produce a symmetrical period change distribution either.

An explanation might be found by considering the sinusoidal period changes clearly seen in some of the $\mathrm{O}-\mathrm{C}$ diagrams with long baselines e.g. J165649. In Fig. 4, if we only had the first five or six years of observations, the data would be well-fitted by a quadratic opening upwards, and we should conclude that the system was undergoing rapid period increase; conversely, if we only had the last five years of data, the diagram would support a quadratic opening downwards, and the system would appear

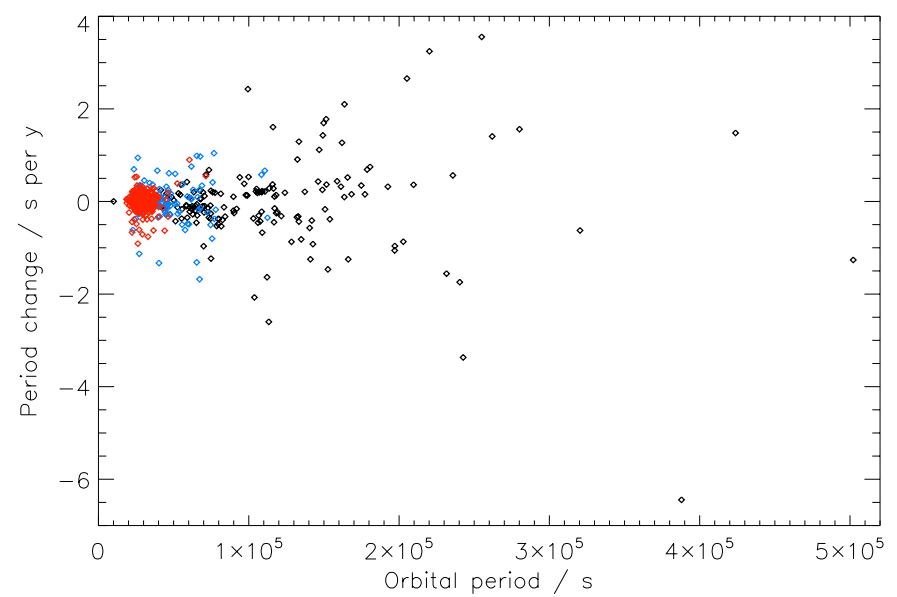

Fig. 7. Period change measurements for 639 distinct sources exhibiting apparent quadratic variation in their $\mathrm{O}-\mathrm{C}$ diagrams, plotted against their orbital periods. EW-type binaries are shown in red, EB-types in blue, and EA-types in black.

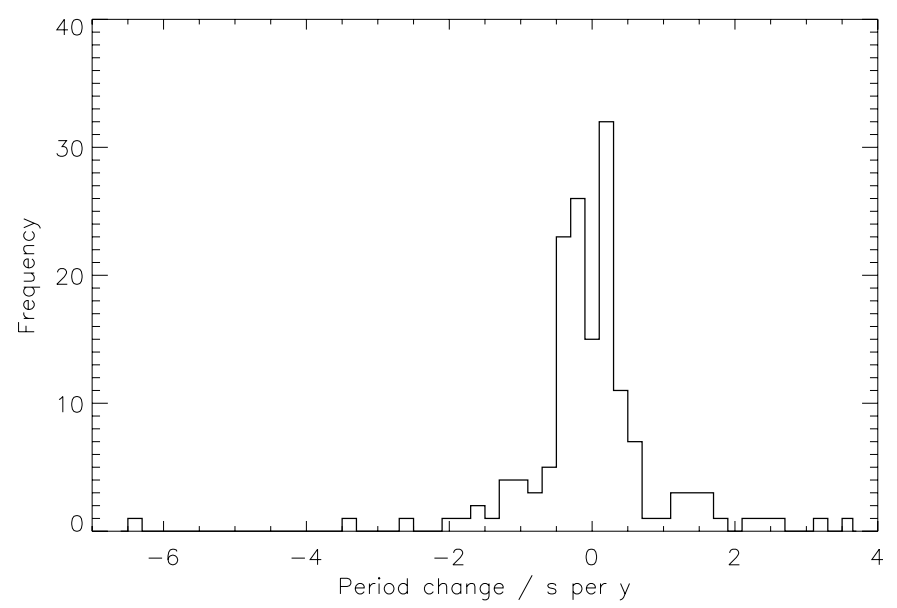

Fig. 8. Period change distribution for EA-type eclipsing binaries.

to be undergoing steady period decrease. Given this, it seems plausible that many of the apparent quadratic changes detected here would prove to be part of longer-term sinusoidal variations if we continued to observe the systems. If the majority of period changes in our data set are actually short sections of sinusoidal variations, this would neatly explain the symmetric distributions seen in Figs. 8 to 10, since the sections would be equally likely to be drawn from any part of the underlying sinusoid, implying equal numbers of apparent positive and negative period changes, on average, for a large sample.

There are two plausible causes for such widespread sinusoidal period variations. The Applegate mechanism (Applegate 1992) could produce semi-sinusoidal modulations, of amplitude $\Delta P / P \sim 10^{-5}$ on a time scale of decades, in close binaries containing at least one active, convective star; luminosity variations would also be expected to be observed with the same period as the $\mathrm{O}-\mathrm{C}$ modulation. However, it is unlikely or impossible that this mechanism is responsible for many of the cases seen here, which include widely-separated long-period binaries as well as W UMa-type systems, systems not exhibiting obvious luminosity changes of the correct period (e.g. J165649, Fig. 4), systems exhibiting modulation on quite short time scales (e.g. J161253, Fig. 5) and O-C amplitudes substantially too large (e.g. J051927). The similar shapes of the three distributions varying primarily in scale - also suggest a common underlying 


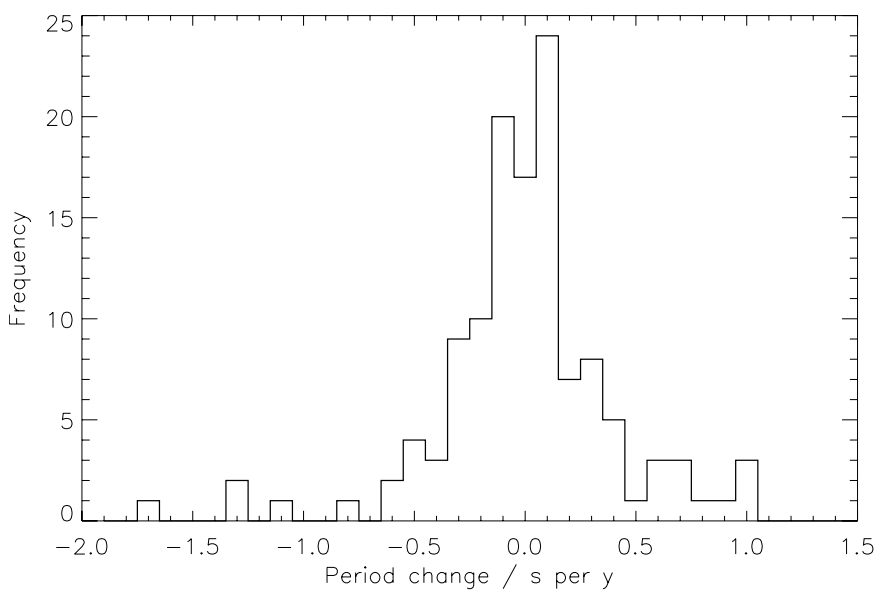

Fig. 9. Period change distribution for EB-type eclipsing binaries.

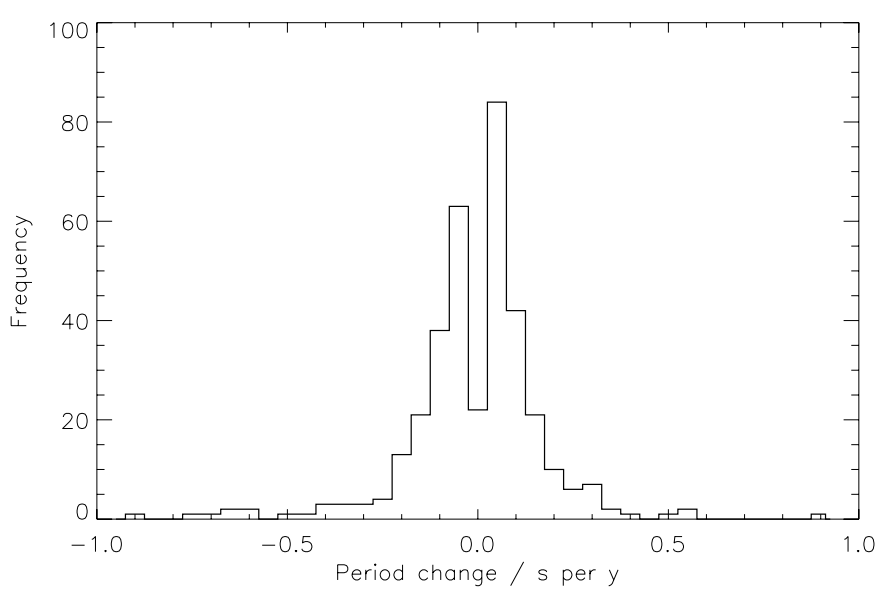

Fig. 10. Period change distribution for EW-type eclipsing binaries.

cause, rather than different mechanisms operating in different types of system.

A more straightforward explanation, which would be applicable to nearly all types of eclipsing systems seen here, would be the influence of a third body inducing sinusoidal period modulation through the Roemer (light travel time) delay and/or the physical delay for a third star in an eccentric or inclined-plane orbit (Rappaport et al. 2013 gives further details of the expected contributions of each effect in systems of different configurations). Further support for this cause is provided by the greater frequency of period changes seen in short-period EW-type systems compared with long-period EBs and EAs (Fig. 7): the modulation amplitude may be expected to be greater, and the modulation period shorter, in closer systems containing low-mass binaries. Moreover, a third star may have actually driven a binary to shorter orbital periods and towards contact configuration through Kozai cycles (Kozai 1962). Additionally, higher-order multiplicity in some eclipsing SuperWASP binaries has been strongly supported by other techniques (Lohr et al. 2013a,b), so such systems are not inherently unlikely.

Estimates of the modulating periods of our samples of objects whose $\mathrm{O}-\mathrm{C}$ diagrams show at least one full cycle of sinusoidal period variation allow us to find approximate ratios of outer to inner orbits, on the assumption that third bodies are present $\left(P_{\mathrm{L}} / P_{\mathrm{S}}\right.$ in terms of Tokovinin $\left.2014 \mathrm{~b}\right)$. The outer orbits have periods between about two and seven years (the upper limit being provided by the time span of SuperWASP archival light curves), giving ratios between 250 and 10000 . These are all well above the minimum ratio for dynamical stability of triple systems, given by Tokovinin as 4.7 , or 47 for systems with high eccentricity of the outer orbit. As is shown in Tokovinin's Fig. 7 (2014b), "there is no typical or preferred period ratio [...] all allowed combinations of periods actually happen", and ratios between $P_{\mathrm{L}} / P_{\mathrm{S}}$ of five and $10^{8}$ are found. There is perhaps a lack of systems with $P_{\mathrm{L}}<10^{3} \mathrm{~d}$ in that figure, but Conroy et al. (2014) have recently identified 236 candidate Kepler close binaries in triple systems, 35 of which have $P_{\mathrm{L}}<700 \mathrm{~d}$. Thus our visibly sinusoidally-varying objects would also be fully consistent with the known period ratios in triples.

In spite of the symmetry of our period change distributions, a few of the objects here exhibiting quadratic variations in their $\mathrm{O}-\mathrm{C}$ diagrams might still be better explained by other factors such as mass transfer or loss, as argued for a selection of 18 known Algols in Erdem \& Öztürk (2014). (We may note that two of their cases exhibited very dramatic period increases of 18.8 and $22.9 \mathrm{~s} \mathrm{yr}^{-1}$ : far greater than any observed here.) There is, however, no reason why a system should not exhibit both mass transfer/loss and third body effects in a single light curve, and Soydugan et al. (2003) claim precisely this for the Algol-type systems S Equ and AB Cas.

If all the period changes measured here were actually associated with third bodies, their frequency within the sample of SuperWASP eclipsing binary candidates would allow us to place a lower limit on the frequency of triples amongst binaries more generally, as around $24 \%$. (Of course, some of these detected period changes probably have other causes, so in a sense it is also an upper limit.) This value lies between Tokovinin's slightly higher figure of $29 \%$ for $\mathrm{F}$ and $\mathrm{G}$ dwarfs (taking into account detection biases), and Rappaport et al.'s estimate of "at least $20 \%$ of all close binaries".

\section{Conclusion}

A neural-net-based catalogue of $\sim 14000$ candidate SuperWASP eclipsing binaries was searched to check their orbital periods and classification, and to search for evidence of period change. Numerous clear cases of quadratic and sinusoidal variation in $\mathrm{O}-\mathrm{C}$ diagrams were observed; interpreting the quadratic variation as sections of longer-period sinusoidal variation would explain the symmetrical period change distributions observed in all three classes of binaries. If this period modulation is caused by third bodies, this allows us to estimate a lower limit for the higher-order multiplicity fraction among local galactic binaries of around $24 \%$, which tallies well with other estimates. In the future, we would hope to confirm some of these candidate triple systems by direct imaging and/or spectroscopic follow-up observations.

Acknowledgements. The WASP project is currently funded and operated by Warwick University and Keele University, and was originally set up by Queen's University Belfast, the Universities of Keele, St. Andrews and Leicester, the Open University, the Isaac Newton Group, the Instituto de Astrofisica de Canarias, the South African Astronomical Observatory and by STFC. This work was supported by the Science and Technology Funding Council and the Open University.

\section{References}

Applegate, J. H. 1992, ApJ, 385, 621

Budding, E., Erdem, A., Çiçek, C., et al. 2004, A\&A, 417, 263

Conroy, K. E., Prsa, A., Stassun, K. G., et al. 2014, AJ, 147, 45

Deschamps, R., Siess, L., Davis, P. J., \& Jorissen, A. 2013, A\&A, 557, A40 
M. E. Lohr et al.: Period changes and multiplicity in SuperWASP binaries

Duchêne, G., \& Kraus, A. 2013, ARA\&A, 51, 269

Eggleton, P. 2006, Evolutionary Processes in Binary and Multiple Stars (Cambridge, UK: Cambridge University Press)

Erdem, A., \& Öztürk, O. 2014, MNRAS, 441, 1166

Hilditch, R. W. 2001, An Introduction to Close Binary Stars (Cambridge, UK: Cambridge University Press)

Ibanoglu, C., Soydugan, F., Soydugan, E., \& Dervisoglu, A. 2006, MNRAS, 373, 435

Koen, C. 2014, MNRAS, 441, 3075

Koo, J.-R., Lee, J. W., Lee, B.-C., et al. 2014, AJ, 147, 104

Kozai, Y. 1962, AJ, 67, 591

Lohr, M. E., Norton, A. J., Kolb, U. C., et al. 2012, A\&A, 542, A124

Lohr, M. E., Norton, A. J., Kolb, U. C., \& Boyd, D. R. S. 2013a, A\&A, 558, A71

Lohr, M. E., Norton, A. J., Kolb, U. C., et al. 2013b, A\&A, 549, A86

Lohr, M. E., Norton, A. J., Anderson, D. R., et al. 2014, A\&A, 566, A128

Michaely, E., \& Perets, H. B. 2014, ApJ, 794, 122
Naoz, S., \& Fabrycky, D. C. 2014, ApJ, 793, 137

Norton, A. J., Wheatley, P. J., West, R. G., et al. 2007, A\&A, 467, 785

Norton, A. J., Payne, S. G., Evans, T., et al. 2011, A\&A, 528, A90

Paczyński, B., Szczygieł, D. M., Pilecki, B., \& Pojmański, G. 2006, MNRAS, 368,1311

Payne, S. G. 2013, Catalogue of SuperWASP variable stars: Ph.D. dissertation (Open University)

Pollacco, D. L., Skillen, I., Cameron, A. C., et al. 2006, PASP, 118, 1407

Rappaport, S., Deck, K., Levine, A., et al. 2013, ApJ, 768, 33

Siess, L., Izzard, R. G., Davis, P. J., \& Deschamps, R. 2013, A\&A, 550, A100

Soydugan, F., Demircan, O., Soydugan, E., \& Ibanoglu, C. 2003, AJ, 126, 393

Stepień, K., \& Gazeas, K. 2012, Acta Astron., 62, 153

Tokovinin, A. 2014a, AJ, 147, 86

Tokovinin, A. 2014b, AJ, 147, 87

van Rensbergen, W., de Greve, J. P., Mennekens, N., Jansen, K., \& de Loore, C. 2011, A\&A, 528, A16 